Gut and Liver, Vol. 10, No. 6, November 2016, pp. 871-872

EDITORIAL

\title{
Role of Periostin in Hepatocellular Carcinoma: The Importance of Tumor Microenvironment
}

\author{
Jung II Lee \\ Department of Internal Medicine, Yonsei University College of Medicine, Seoul, Korea
}

\begin{abstract}
See "The Combination of Periostin Overexpression and Microvascular Invasion Is Related to a Poor Prognosis for Hepatocellular Carcinoma" by Se Young Jang, et al. on page 948, Vol. 10. No. 6, 2016
\end{abstract}

The contribution of the tumor microenvironment in cancer progression is becoming widely recognized. The tumor microenvironment consists not only of cellular components such as immune cells, fibroblasts, and endothelial cells as well as the malignant tumor cells themselves, but also includes noncellular component such as the extracellular matrix (ECM). ${ }^{1}$

The ECM is a complex meshwork made up of both proteins and proteoglycans with covalently attached sugar chains, glycosaminoglycans. Although primary role of ECM may be to provide structural support of an organ, it is also crucial in modulating cell functions by playing a role as a reservoir for growth factors or signaling molecules. ${ }^{2}$ ECM signaling may involve in proliferation, migration and invasion of cancerous cells. In addition collagen fibers of ECM can build migration tracks for tumor cells. At the same time, ECM may work as a barrier, hindering the penetration of immune cells or drugs into the tumor sites.

Periostin, also known as osteoblast-specific factor 2, is initially identified as a cell adhesion protein in mouse osteoblast cell line. It is a secreted N-glycoprotein and is reported to interact with other ECM proteins such as collagen I, fibronectin, and tenascin C. Meanwhile, several recent studies demonstrated enhanced expression of periostin in breast, colon, lung, and pancreas cancer. ${ }^{3-6}$ Moreover, high serum periostin level was detected in patients with advanced lung and breast cancer accompanied by metastasis of the disease. ${ }^{5,6}$

Jang et al. ${ }^{7}$ reported that high periostin expression was related with decreased survival and increased recurrence in hepatocellular carcinoma (HCC). They suggested that pathological overexpression of periostin, in combination with existence of microvascular invasion could be a useful prognostic marker for HCC. Although existence of microvascular invasion is widely accepted as an important predictor recurrence after the curative treatment, usefulness of serum and pathologic periostin expression in HCC still needs some validations. However, the results from the study by Jang et al. ${ }^{7}$ are in accordance with the previous studies. A study which included 56 HCC patients reported that elevated serum periostin levels indicated decreased diseasefree survival (DFS) and overall survival (OS). ${ }^{8}$ Another study that evaluated pathological expression of periostin in 71 HCC patients also showed that high periostin expression was associated with compromised DFS as well as OS. ${ }^{9}$

Periostin, ECM protein, seems to contribute in liver fibrosis. Periostin expression was significantly upregulated in carbon tetrachloride and bile duct ligation induced liver cirrhosis. ${ }^{10}$ On the other hands, periostin deficient mice demonstrated attenuated liver fibrosis. In addition, periostin downregulation resulted in ameliorated hepatic stellate activation and proliferation, which might be directly related with liver fibrosis. ${ }^{11}$ Liver fibrosis and cirrhosis are one of the most important risk factors for HCC occurrence. HCC is very frequently accompanied by underlying liver fibrosis and cirrhosis and there is increased chance that expression of periostin in HCC might be associated with underling liver condition.

The study by Jang et al. ${ }^{7}$ did not comment on the existence of liver cirrhosis in the recruited patients that were tested for histological periostin expression in HCC. However, previous study by Lv et al. ${ }^{8}$ reported increased serum periostin level in patient with liver cirrhosis compared with that of the healthy volunteers. The study also compared serum periostin levels between patients

Correspondence to: Jung Il Lee

Department of Internal Medicine, Yonsei University College of Medicine, 211 Eonju-ro, Gangnam-gu, Seoul 06273, Korea

Tel: +82-2-2019-3310, Fax: +82-2-3463-3882, E-mail: mdflorence@yuhs.ac

pISSN 1976-2283 eISSN 2005-1212 https://doi.org/10.5009/gnl16429

@ This is an Open Access article distributed under the terms of the Creative Commons Attribution Non-Commercial License (http://creativecommons.org/licenses/by-nc/4.0) which permits unrestricted non-commercial use, distribution, and reproduction in any medium, provided the original work is properly cited. 
with liver cirrhosis and HCC, and demonstrated the higher levels in HCC patients. It would have been interesting if pathologic periostin expression in HCC have been investigated not only in tumor sites but also in the corresponding nontumor sites. This comparison might give some clues for periostin expression in HCC and its relation with the background liver fibrosis/cirrhosis.

Tissue and serum periostin expression have been extensively studied in advanced breast cancer to test its qualification as a tumor marker. As in HCC, the usefulness of tissue periostin as a prognostic marker for recurrence and metastasis should further be examined in other studies. In addition, role of serum periostin level, which is definitely more accessible means of testing, may need to be investigated and validated in other studies.

\section{CONFLICTS OF INTEREST}

No potential conflict of interest relevant to this article was reported.

\section{REFERENCES}

1. Venning FA, Wullkopf L, Erler JT. Targeting ECM disrupts cancer progression. Front Oncol 2015;5:224.

2. Hynes R0. The extracellular matrix: not just pretty fibrils. Science 2009;326:1216-1219.

3. Bao S, Ouyang G, Bai X, et al. Periostin potently promotes metastatic growth of colon cancer by augmenting cell survival via the Akt/PKB pathway. Cancer Cell 2004;5:329-339.
4. Fukushima N, Kikuchi Y, Nishiyama T, Kudo A, Fukayama M. Periostin deposition in the stroma of invasive and intraductal neoplasms of the pancreas. Mod Pathol 2008;21:1044-1053.

5. Sasaki H, Dai M, Auclair D, et al. Serum level of the periostin, a homologue of an insect cell adhesion molecule, as a prognostic marker in nonsmall cell lung carcinomas. Cancer 2001;92:843-848.

6. Contié S, Voorzanger-Rousselot N, Litvin J, Clézardin P, Garnero P. Increased expression and serum levels of the stromal cell-secreted protein periostin in breast cancer bone metastases. Int $\mathrm{J}$ Cancer 2011;128:352-360.

7. Jang SY, Park SY, Lee HW, et al. The combination of periostin overexpression and microvascular invasion is related to a poor prognosis for hepatocellular carcinoma. Gut Liver 2016;10:948954.

8. Lv Y, Wang W, Jia WD, et al. High preoparative levels of serum periostin are associated with poor prognosis in patients with hepatocellular carcinoma after hepatectomy. Eur J Surg Oncol 2013;39:1129-1135.

9. Lv Y, Wang W, Jia WD, et al. High-level expression of periostin is closely related to metastatic potential and poor prognosis of hepatocellular carcinoma. Med Oncol 2013;30:385.

10. Huang Y, Liu W, Xiao H, et al. Matricellular protein periostin contributes to hepatic inflammation and fibrosis. Am J Pathol 2015;185:786-797.

11. Hong L, Shejiao D, Fenrong C, Gang Z, Lei D. Periostin down-regulation attenuates the pro-fibrogenic response of hepatic stellate cells induced by TGF-beta1. J Cell Mol Med 2015;19:2462-2468. 